\title{
Perfil socioeconômico dos acadêmicos de Odontologia usuários de álcool e tabaco
}

Gisele Ristow Montes*; Judith Angélica Gonzales*; Fernanda Morais Ferreira**; Luciana Reichert da Silva Assunção***; Danielle Medeiros Veiga Bonotto*; Fabian Calixto Fraiz****

$\begin{array}{ll}\text { * } & \text { Cirurgiã-Dentista, Mestre em } \\ & \text { Odontologia pelo Programa de Pós- } \\ \text { Graduação em Odontologia da } & \\ \text { Universidade Federal do Paraná (UFPR). } & \text { Professora Adjunta do Departamento de } \\ \text { Odontopediatria e Ortodontia da } & \\ & \text { Universidade Federal de Minas Gerais } \\ & \text { (UFMG). } \\ \text { Professora Adjunta do Departamento de } \\ \text { Estomatologia da Universidade Federal } \\ \text { do Paraná (UFPR). } \\ \text { Professor Titular do Departamento de } \\ \text { Estomatologia da Universidade Federal } \\ \text { do Paraná (UFPR). }\end{array}$

\section{RESUMO}

O objetivo deste estudo foi avaliar o uso de álcool e tabaco entre os acadêmicos de odontologia. Foi realizado um estudo transversal censitário com 300 acadêmicos de odontologia da Universidade Federal do Paraná (UFPR). As informações sobre o perfil socioeconômico, assim como acerca do uso de álcool e tabaco foram coletadas por meio de um questionário especificamente desenvolvido para essa pesquisa. A população estudada era na maioria do sexo feminino e com idade média de $21 \pm 2,5$ anos, sendo que $51,3 \%$ e $8,4 \%$ usavam álcool e tabaco, respectivamente. Estudantes do sexo masculino apresentaram maior prevalência de uso de álcool $(\mathrm{RP}=1,58$; IC 95\%: 1,09-2,28) e tabaco (RP=1,72; IC 95\%: 1,102,70). Maior prevalência de uso de álcool $(\mathrm{RP}=1,70 ; \quad \mathrm{IC}$ 95\%: 1,30-2,21) e tabaco $(\mathrm{RP}=1,50$; IC 95\%: 1,10-2,05) foi observada entre os estudantes de classes econômicas mais altas, quando comparados aos menos favorecidos economicamente. Os estudantes que não moravam com a família apresentaram prevalência de consumo de álcool $70 \%$ maior do que os que residiam com a família $(\mathrm{RP}=1,70$; IC 95\%: 1,10-2,65). O uso de tabaco não diferiu em função da moradia. Conclui-se que os estudantes de odontologia da UFPR que são usuários de álcool e tabaco apresentam especificidade social e econômica, assim esforços devem ser direcionados para a conscientização e proteção desse grupo.

Descritores: Estudantes de Odontologia. Hábito de Fumar. Bebidas Alcoólicas.

\section{INTRODUÇÃO}

A prevalência do consumo de substâncias psicoativas em universitários brasileiros é alta, e o álcool e tabaco estão entre as mais utilizadas ${ }^{1-8}$. Além dos problemas de 
saúde diretamente relacionados ao hábito de beber e fumar, também tem sido relatado o uso de substâncias ilícitas associadas ao consumo de álcool e tabaco entre universitários de odontologia ${ }^{6,9,10}$.

Os fatores apontados como responsáveis pelo consumo excessivo de álcool e tabaco na fase universitária incluem a transição do ensino médio para a universidade com o estabelecimento de novas amizades e de uma atividade social intensa ${ }^{1,10-12}$. As tensões emocionais surgidas da pressão por bons resultados na vida acadêmica e da constante competição para uma vida profissional futura bem-sucedida podem induzir o uso dessas substâncias, na busca da diminuição do estresse e de situações ansiogênicas ${ }^{12,13}$.

Além de diminuir o rendimento escolar, o abuso de álcool eleva os índices de acidentes de trânsito, de violência urbana, assim como estimula comportamentos de risco como a prática sexual desprotegida ${ }^{3,5,10,14}$. Entre adolescentes, o consumo de álcool aumenta o risco de depressão e ansiedade ${ }^{15}$ e também está associado ao desenvolvimento de alcoolismo, problemas cognitivos, comportamentais e emocionais ${ }^{16}$.

Apesar da utilização de tabaco estar diminuindo nos últimos anos, principalmente nos países mais desenvolvidos, estima-se que, a cada ano, ainda morram cerca de 4 milhões de pessoas em todo o mundo de doenças relacionadas a esse hábito ${ }^{17}$. Adolescentes permanecem como um grupo vulnerável à exposição de propagandas da indústria do tabaco $^{18}$ e mesmo com as ações antifumo adotadas pelo Brasil, sua utilização entre jovens ainda é motivo de preocupação.

$\mathrm{O}$ estudante de odontologia tem amplo acesso às informações sobre as consequências da utilização de álcool e de tabaco, já que as mesmas estão associadas a diversos agravos em saúde bucal, desde o impacto negativo na estética do sorriso até a maior predisposição ao câncer bucal ${ }^{19}$. O tabagismo é o principal fator de risco para carcinoma espinocelular de cabeça e pescoço ${ }^{20}$ e $75 \%$ dos casos de câncer nessas regiões são atribuídos à combinação dos hábitos de fumar e beber ${ }^{21}$.

Embora fosse esperado que acadêmicos da área de saúde apresentassem níveis de conhecimento e conscientização capazes de inibir o uso de drogas lícitas e ilícitas, diversos estudos indicam que, mesmo neste grupo, o uso dessas substâncias é alto ${ }^{6,10,22}$, demonstrando que, para preveni-lo, o simples contato com a informação pode não ser suficiente.

Esta pesquisa teve como objetivo principal conhecer, em uma população de estudantes de odontologia, como esses hábitos são influenciados pelo gênero e condições socioeconômicas. Entender essas relações permitirá a definição de ações preventivas efetivas e factíveis voltadas para grupos que, embora tenham acesso ao conhecimento dos efeitos adversos, mantêm-se vulneráveis a adoção desses hábitos.

\section{MÉTODOS}

Um estudo transversal censitário foi conduzido com 375 alunos de graduação do Curso de Odontologia da Universidade Federal do Paraná (UFPR), Brasil, em 2011, sendo que 307 alunos concordaram em participar. Assim a taxa de resposta foi de $81,9 \%$. Desses, sete questionários foram excluídos por preenchimento incompleto, totalizando 300 participantes.

As características sociodemográficas, de uso de álcool e tabaco foram coletadas por meio de um questionário especificamente desenvolvido para essa pesquisa. O instrumento foi testado inicialmente com 15 estudantes de graduação de outro curso da área de saúde, na 
mesma instituição, para reestruturação e verificação da consistência das questões. O formulário incluiu questões referentes ao gênero, idade, moradia (mora com os pais, parentes, cônjuge ou mora sozinho, em república, pensão), estágio de formação acadêmica (semestre do curso), grau de instrução do provedor (em nove categorias variando de "sem estudo" a "superior completo"), consumo de tabaco e de álcool (ambos categorizados em nunca, raramente, às vezes, quase sempre ou sempre). Para a classificação econômica foi aplicado um instrumento da Associação Brasileira de Empresas de Pesquisa - ABEP (2010) ${ }^{23}$, Critério de Classificação Econômica Brasil (CCEB) amplamente difundido. O CCEB é um instrumento de segmentação econômica que utiliza o levantamento de características domiciliares (presença e quantidade de alguns itens domiciliares de conforto e grau de escolaridade do chefe de família) para diferenciar a população. Este critério atribui pontos em função de cada característica domiciliar, os quais são somados. É feita, então, uma correspondência entre faixas de pontuação do critério e estratos de classificação econômica definidos por A1, A2, B1, B2, C, D, E23.

Os questionários foram aplicados em sala de aula por uma mesma pesquisadora (JAGS) que esteve presente para acompanhar o processo e resolver dúvidas. Foram dadas explicações referentes à pesquisa e após a concordância iniciou-se o processo de coleta de dados na seguinte sequência: formulário sóciodemográfico, uso de tabaco e álcool e classificação econômica. Para garantir a privacidade das informações, cada aluno preencheu os dados individualmente, o que garantiu anonimato.

Para as análises descritivas e inferenciais, as variáveis dependentes consumo de álcool e de tabaco foram dicotomizadas em "Não" (nunca ou raramente) ou "Sim" (às vezes, quase sempre ou sempre). As variáveis independentes foram categorizadas em gênero ("masculino" ou "feminino"); moradia com a família ("Sim - mora com os pais, parentes, /cônjuge" ou "Não - mora sozinho, em republica, pensão"), classificação econômica ("A1,A2" ou "B1,B2,C,D,E”), grau de instrução do provedor ("mais que 8 anos de educação formal" ou "igual ou menos que 8 anos de educação formal"), ciclo de formação acadêmica ("Básico - até o $4^{\circ}$ semestre" ou "Profissional - a partir do $5^{\circ}$ semestre").

Foram utilizadas análises bivariadas para verificar a diferença na prevalência de consumo de álcool e tabaco em função das variáveis independentes categóricas por meio do teste Qui-quadrado e calculada a Razão de Prevalência e seu respectivo intervalo de confiança (95\%). A relação da variável numérica discreta "idade" e as categorias de interesse foi analisada por meio do teste não paramétrico de Mann-Whitney. Para a avaliação da associação entre o consumo de álcool e tabaco foi utilizado o teste de Quiquadrado. Os dados foram analisados com auxílio do programa Statistical Package for the Social Science version 20 (SPSS). O nível de significância adotado em todas as análises foi de $5 \%$.

Este estudo recebeu aprovação do Comitê de Ética em Pesquisa em Seres Humanos da UFPR e foi conduzido de acordo com a declaração de Helsinki (CAAE: 0061.0.91.000-11). Os estudantes ou seu responsável legal assinaram um termo de consentimento livre e esclarecido, autorizando a participação no estudo.

\section{RESULTADOS}

A média de idade dos participantes foi 
de 21 2 ,5anos (amplitude: 17-34) com predomínio da faixa etária até 23 anos e do sexo feminino. A grande maioria dos provedores apresentavam mais de 8 anos de estudo formal. O consumo de álcool e tabaco entre os estudantes foi de $51,3 \%$ e $8,3 \%$, respectivamente (Tabela 1 ).
A prevalência de consumo de álcool e tabaco entre os estudantes das classes econômicas A1 e A2 foi, respectivamente, 1,70 $(P<0,001)$ e $1,50(P=0,036)$ vezes a prevalência daqueles de classes econômicas menos favorecidas (Tabela 2).

Tabela 1 - Características socioeconômicas, formação acadêmica e consumo de álcool e tabaco pelos estudantes de odontologia da UFPR.

\begin{tabular}{|c|c|c|}
\hline Variável & $\mathbf{N}$ & $\%$ \\
\hline \multicolumn{3}{|l|}{ Gênero } \\
\hline Masculino & 88 & 29,3 \\
\hline Feminino & 212 & 70,7 \\
\hline \multicolumn{3}{|l|}{ Mora com a família } \\
\hline Sim & 233 & 77,7 \\
\hline Não & 67 & 22,3 \\
\hline \multicolumn{3}{|l|}{ Classificação Econômica } \\
\hline $\mathrm{A} 1, \mathrm{~A} 2$ & 138 & 46,0 \\
\hline B1, B2 & 140 & 46,7 \\
\hline$C, D$ e $E$ & 18 & 6,0 \\
\hline Não informou & 4 & 1,3 \\
\hline \multicolumn{3}{|l|}{ Anos de estudo do Provedor } \\
\hline Mais que 8 anos & 280 & 93,3 \\
\hline Até 8 anos & 20 & 6,7 \\
\hline \multicolumn{3}{|l|}{ Formação acadêmica (ciclo) } \\
\hline Básico ( $1^{\circ}$ a $4^{\circ}$ período $)$ & 151 & 50,3 \\
\hline Profissionalizante $\left(5^{\circ}\right.$ a $9^{\circ}$ período $)$ & 149 & 49,7 \\
\hline \multicolumn{3}{|l|}{ Consumo de Álcool } \\
\hline Não & 146 & 48,7 \\
\hline Sim & 154 & 51,3 \\
\hline \multicolumn{3}{|l|}{ Consumo de Tabaco } \\
\hline Não & 273 & 91,0 \\
\hline Sim & 25 & 8,3 \\
\hline
\end{tabular}

Valores inferiores a 300 se devem a não resposta de algum item. 
Tabela 2 - Associação entre consumo de álcool e tabaco de acordo com as características socioeconômicas dos estudantes de odontologia da UFPR.

\begin{tabular}{|c|c|c|c|c|c|c|c|c|}
\hline \multirow[b]{3}{*}{ Gênero } & \multicolumn{4}{|c|}{ Álcool } & \multicolumn{4}{|c|}{ Tabaco } \\
\hline & $\begin{array}{l}\text { Não } \\
\mathrm{n}(\%)\end{array}$ & $\begin{array}{l}\mathrm{Sim} \\
\mathrm{n}(\%)\end{array}$ & $p^{*}$ & $\begin{array}{c}\mathrm{RP} \\
(\mathrm{IC} 95 \%)\end{array}$ & $\begin{array}{l}\text { Não } \\
\text { n(\%) }\end{array}$ & $\begin{array}{l}\mathrm{Sim} \\
\mathrm{n}(\%)\end{array}$ & $p^{*}$ & $\begin{array}{c}\mathrm{RP} \\
(\mathrm{IC} 95 \%)\end{array}$ \\
\hline & & & & & & & & \\
\hline Masculino & $33(37)$ & $55(63)$ & 0,013 & $\begin{array}{c}1,58 \\
(1,09-2,28)\end{array}$ & $76(86)$ & $12(14)$ & $\mathbf{0 , 0 3 4}$ & $\begin{array}{c}1,72 \\
(1,10-2,70)\end{array}$ \\
\hline Feminino & $113(53)$ & $99(47)$ & & 1 & 197(94) & $13(6)$ & & 1 \\
\hline \multicolumn{9}{|c|}{ Classificação Econômica } \\
\hline $\mathrm{A} 1, \mathrm{~A} 2$ & $49(35)$ & $89(65)$ & $<0,001$ & $\begin{array}{c}1,70 \\
(1,30-2,21)\end{array}$ & $120(88)$ & $16(12)$ & $\mathbf{0 , 0 3 6}$ & $\begin{array}{c}1,50 \\
(1,10-2,05)\end{array}$ \\
\hline B1 a E & $94(59)$ & $64(41)$ & & 1 & $150(95)$ & $8(5)$ & & 1 \\
\hline \multicolumn{9}{|c|}{ Mora com família } \\
\hline Não & $24(36)$ & $43(64)$ & $\mathbf{0 , 0 1 7}$ & $\begin{array}{c}1,70 \\
(1,10-2,65)\end{array}$ & 58(89) & $7(11)$ & 0,434 & $\begin{array}{c}1,32 \\
(0,67-2,57)\end{array}$ \\
\hline Sim & $122(52)$ & $111(48)$ & & 1 & $215(92)$ & $18(8)$ & & 1 \\
\hline \multicolumn{9}{|c|}{ Formação acadêmica (ciclo) } \\
\hline Profissional & $72(48)$ & $77(52)$ & 0,906 & $\begin{array}{c}1,01 \\
(0,81-1,27)\end{array}$ & 133(90) & $14(10)$ & 0,486 & $\begin{array}{c}1,15 \\
(0,79-1,66)\end{array}$ \\
\hline Básico & 74(49) & $77(51)$ & & 1 & $140(93)$ & 11(7) & & 1 \\
\hline
\end{tabular}

* Teste Qui- Quadrado

$\mathrm{RP}=$ Razão de prevalência

IC=Intervalo de confiança

Resultados significantes ao nível de 5\% marcados em negrito.

Tabela 3 - Média de idade dos estudantes de Odontologia da UFPR de acordo com o uso de tabaco ou álcool.

\begin{tabular}{lcccc}
\hline & Álcool & & Tabaco & \\
& & & & \\
& Média (DP) & $p^{*}$ & Média (DP) & $p^{*}$ \\
\cline { 2 - 5 } NÃO & $21,3(2,9)$ & & $21,1(2,5)$ & \\
SIM & $21,1(2,2)$ & 0,889 & $\mathbf{0 , 0 1 5}$ \\
\hline
\end{tabular}

*Teste Mann-Whitney

$\mathrm{DP}=$ desvio padrão

Resultados significantes ao nível de 5\% marcados em negrito. 
Tabela 4 - Relação entre o consumo de álcool e de tabaco pelos estudantes de Odontologia da UFPR.

\begin{tabular}{lccccc}
\hline & & \multicolumn{2}{c}{ Tabaco } & Total & $\boldsymbol{p}^{*}$ \\
\hline & & Não & Sim & & \\
\hline \multirow{2}{*}{ Álcool } & Não & $143(48)$ & N $(\%)$ & & \\
\multirow{2}{*}{ Total } & Sim & $130(43,6)$ & $22(7,4)$ & $152(51)$ & $<0,001$ \\
\hline
\end{tabular}

* Teste Qui- Quadrado

Resultados significantes ao nível de 5\% marcados em negrito.

Valores inferiores a 300 se devem a não resposta de algum item.

Aqueles que moravam sozinhos, em república ou pensão apresentaram prevalência de utilização de álcool maior do que os que residiam com os pais, parentes e cônjuges ( $R P=1,70$; IC 95\%: 1,10-2,65). No entanto, a prevalência de consumo de tabaco não foi diferente em função do tipo de moradia (Tabela 2).

A prevalência de consumo das substâncias pesquisadas não foi diferente em função do ciclo de formação acadêmica (Tabela 2).

A idade não diferiu entre os estudantes que consumiram ou não álcool $(P=0,889)$. Porém o grupo de estudantes que consumia tabaco era mais velho do que os que não consumiam ( $P=0,015$; Tabela 3$)$.

Pode-se observar associação estatisticamente significante entre o consumo de álcool e de tabaco (Tabela 4), sendo que o consumo concomitante das duas substâncias foi observado em $7,4 \%$ do total da população de estudo (Tabela 4).

\section{DISCUSSÃO}

Apesar do consumo de álcool e tabaco entre os acadêmicos de odontologia avaliados ter sido mais baixo do que o encontrado em levantamento nacional com estudantes universitários brasileiros $^{7}$, esperava-se que a prevalência de uso dessas substancias fosse ainda menor, já que durante a graduação em odontologia os acadêmicos têm grande disponibilidade de informação sobre os riscos de tabagismo e de consumo de álcool.

Embora tenham conhecimento dos efeitos prejudiciais do uso de bebidas alcoólicas, o clima festivo universitário e a busca pela aceitação no grupo são fatores que podem estimular o consumo ${ }^{12}$. Outros estudos com universitários também têm encontrado um consumo alto de álcool ${ }^{1,12}$, incluindo estudantes da área de saúde ${ }^{1,8,11}$ e odontológica ${ }^{6,9,10}$.

Em uma perspectiva global, o consumo de tabaco entre estudantes de odontologia têm diminuído ${ }^{24}$. Nesse estudo, menos que um décimo dos acadêmicos relataram uso de tabaco, sendo que a grande maioria desses utilizava concomitantemente álcool. GranvilleGarcia et al. ${ }^{2}$ encontraram que universitários brasileiros da área de saúde que consumiam álcool apresentavam 3,71 mais chance de fumar do que aqueles que não o consumiam.

Alguns estudos avaliaram a opinião dos universitários em relação a capacidade e pretensão de cessar o hábito de fumar ${ }^{25}$ e de beber ${ }^{13}$, tendo sido observado que grande parte dos universitários subestimavam sua dependência e tinham a convicção que seriam capazes de controlar os eventuais problemas surgidos pelo uso indevido de álcool, tabaco e outras drogas. Outra característica importante observada foi a convivência involuntária de estudantes não tabagistas com os tabagistas, 
tornando assim um ambiente favorável a uso do tabaco $^{25}$.

A utilização de substâncias ilícitas também tem sido associada ao hábito de beber e fumar entre universitários de odontologia ${ }^{6,10}$. É provável que os fatores que estimulam o consumo de substâncias prejudiciais à saúde lícitas ou ilícitas sejam os mesmos, o que pode levar ao uso concomitante e, consequentemente, à soma de impactos adversos. Segundo Horta et al. ${ }^{26}$, o uso de substâncias psicoativas possui um efeito multiplicador, em que o consumo de uma substância aumenta o risco de consumo de outras.

Nesta pesquisa, os estudantes do sexo masculino apresentaram prevalência maior de utilização de álcool e tabaco. Outras pesquisas evidenciaram consumo maior de álcool ${ }^{2,7,27} \mathrm{e}$ tabaco $^{2,10,23}$ entre homens, embora nem todos os trabalhos indiquem diferenças entre os sexos ${ }^{28}$.

Estudo prévio demonstrou que universitários brasileiros do sexo masculino apresentaram expectativas maiores de que o álcool promova transformações globais positivas e de que melhore o desempenho sexual $^{26}$. O consumo de tabaco pelos homens também se apresentou associado ao estereótipo social de masculinidade e o seu consumo diário foi relacionado à necessidade de aceitação e aprovação social ${ }^{29}$.

O presente estudo evidenciou associação positiva entre o uso de álcool e tabaco com a classe econômica, sendo que os estudantes mais privilegiados financeiramente apresentaram maior consumo dessas substâncias. Determinantes econômicos e culturais podem estar relacionados a uma profusão de festas e ao preço de bebidas alcoólicas $^{4,28}$.

Diferenças na prevalência do uso de cigarros e derivados do tabaco foram encontradas em função da renda, país e região entre adolescentes de 32 países ${ }^{30}$. Nos países de alta renda per capita a utilização de tabaco foi maior quando comparado aos países de baixa e média renda. Tais diferenças foram atribuídas às influências culturais, religiosas, ao acesso a diferentes tipos de produtos do tabaco, às estratégias governamentais nacionais e locais de controle e às propagandas da indústria do tabaco $^{30}$.

Estudantes que moravam sozinhos, em pensão ou república apresentaram consumo maior de álcool do que aqueles que moravam com os pais ou cônjuges. Provavelmente esse comportamento está relacionado à maior liberdade dos estudantes que moram sem a presença dos pais e pela necessidade maior de socialização, já que ausência de companhia familiar em casa, o desenvolvimento da autonomia e as demandas universitárias são situações ansiogênicas e estimuladoras das relações interpessoais, as quais podem favorecer o consumo de álcool ${ }^{14}$. Em uma amostra representativa de ingressantes na Universidade Federal de Pelotas foi observado que estudantes que moravam com amigos apresentavam maior consumo de álcool ${ }^{28}$. No entanto, morar com os pais não influenciou o consumo de tabaco. Esse aspecto pode demonstrar o simples desconhecimento dos pais, já que os efeitos imediatos do uso de tabaco são menos visíveis do que o consumo de álcool. Pesquisas posteriores são necessárias para avaliar mais profundamente essa diferença entre o uso de álcool e tabaco.

No presente estudo, não houve diferenças significativas em relação ao consumo de álcool e tabaco e o estágio de formação acadêmica, no entanto, o grupo de estudantes que consumia tabaco era mais velho do que os que não consumiam, confirmando uma tendência linear também apontada no estudo com estudantes da Universidade Federal de Pelotas ${ }^{28}$. A relação entre consumo de álcool e a idade não foi observada. Muitos estudos afirmam que inicia-se cedo o uso do tabaco $^{2,18,30}$ e álcool ${ }^{5,22}$, sendo que esse comportamento pode ter sido adquirido antes do ingresso na universidade.

Para o estabelecimento de estratégias com maior penetração e efetividade é necessário conhecer as características dos grupos mais vulneráveis. No entanto, deve-se reconhecer que generalizações são 
inadequadas, já que o consumo de álcool e tabaco é muito influenciado por especificidades culturais e sociais.

As diretrizes curriculares brasileiras orientam que o estudante de odontologia deverá estar apto a desenvolver ações de prevenção, promoção, proteção e reabilitação da saúde, tanto em nível individual quanto coletivo e dentre as futuras responsabilidades profissionais dos estudantes da área da saúde estão as medidas de orientação e prevenção do consumo de tabaco e álcool.

Assim, reconhecendo que uma parcela desses acadêmicos permanece vulnerável ao consumo dessas substâncias, esforços devem ser direcionados para conscientização e proteção desse grupo. A inclusão curricular de conteúdos e estratégias pedagógicas mais específicas sobre a prevenção e a interrupção do uso de tabaco é fundamental para que a próxima geração de dentistas tenha capacidade de interferir na diminuição do uso de tabaco. $\mathrm{O}$ mesmo deve ser previsto para o álcool.

\section{CONCLUSÃO}

Conclui-se que os estudantes de odontologia da UFPR que são usuários de álcool e tabaco apresentam especificidade social e econômica. Assim esforços devem ser direcionados para a conscientização e proteção desse grupo.

\section{AGRADECIMENTOS:}

Este estudo foi financiado pela Coordenação de Aperfeiçoamento de Pessoal de Nível Superior (Capes), Ministério da Educação, Brasil.

\section{REFERÊNCIAS}

1. Granville-Chapman J, Yu K, White PD. A follow-up survey of alcohol consumption and knowledge in medical students. Alcohol \& Alcoholism 2001;36:540-3.
2. Granville-Garcia AF, Sarmento DJS, Santos JA, Pinto TA, Sousa RV, Cavalcanti AL. Smoking among undergraduate students in the area of health. Ciênc Saúde Coletiva 2012;17(2): 389-96.

3. Nemer ASA, Fausto MA, SilvaFonseca VA, Ciomei MH, Quintaes KD. Padrão de consumo de bebidas alcoólicas e desempenho acadêmico entre universitário. Rev Psiq Clín 2013;40(2):65-70.

4. Silva LVER, Malbergier A, Stempliuk VA, Andrade AG. Fatores associados ao consumo de álcool e drogas entre estudantes universitários. Rev Saúde Pública 2006;40(2):280-8.

5. Wagner GA, Andrade AG. Uso de álcool, tabaco e outras drogas entre estudantes universitários brasileiros. Rev Psiq Clín 2008;35(1):48-54.

6. Teixeira RF, Souza RS, Buaiz V, Siqueira MM. Uso de substâncias psicoativas entre estudantes de odontologia da Universidade Federal do Espírito Santo. Ciênc Saúde Coletiva 2010;15(3):655-62.

7. Brasil. Presidência da República. Secretaria Nacional de Políticas sobre Drogas. I Levantamento Nacional sobre o Uso de Álcool, Tabaco e Outras Drogas entre Universitários das 27 Capitais Brasileiras / Secretaria Nacional de Políticas sobre Drogas; GREA/IPQ-HCFMUSP. Org: Andrade AG, Duarte PCAV, Oliveira LG. Brasília: SENAD; 2010. 284 p.

8. Santos MFVF, Pereira DS, Siqueira MM. Uso de álcool e tabaco entre estudantes de Psicologia da Universidade Federal do Espírito Santo. J Bras Psiquiatr 2013;62(1):22-30.

9. Keshavarz H, Jafari A, Khami MR, Virtanen JI. Passive smoking and attitudes towards tobacco control programs among Iranian dental students. Asian Pac J Cancer Prev 2013;14:3635-9. 
10. Underwood B, Fox K. A survey of alcohol and drug use among UK based dental undergraduates. $\mathrm{Br}$ Den $\mathrm{J}$ 2000;189(6):314-7.

11. Franca LR, Dautzenberg B, Falissard B, Reynaud M. Peer substance use overestimation among French university students: a cross-sectional survey. BMC Public Health 2010;10:169. Disponível em: http:// bmcpublichealth.biomedcentral.com /articles/10.1186/1471-2458-10169.

12. Tavolacci MP, Ladner J, Grigioni S, Richard, Villet H, Dechelotte P. Prevalence and association of perceived stress, substance use and behavioral addictions: a cross-sectional study among university students in France, 2009-2011. BMC Public Heath. 2013;13:724. Disponível em: http://bmcpublichealth.biomedcentr al.com/articles/10.1186/14712458-13-724.

13. Nunes JM, Campolina LR, Vieira MA, Caldeira AP. Consumo de bebidas alcoólicas e práticas do binge drinking entre acadêmicos da área da saúde. Rev Psiq Clín 2012;39:94-9.

14. Miller JW, Naimi TS, Brewer RD, Jones SE. Binge drinking and associated health risk behaviors among high school. Pediatrics 2007;119(1):7685.

15. Balogun O, Koyanagi A, Stickley A, Gilmour S, Shibuya K. Alcohol consumption and psychological distress in adolescents: a multi country study. J Adolesc Health 2014;54 (2):228-34.

16. Mason WA, Spoth RL. Sequence of alcohol involvement from early onset to young adult alcohol abuse: differential predictors and moderation by familyfocused preventive in-tervention. Addiction 2012;107(12): 2137-48.

17. World Health Organization. Confronting the epidemic: a global agenda for tobacco control research.
1999. 2014;12. Disponível em: http:// www.who.int/tobacco/research/en/ print.html.

18. Dube SR, Arrazola RA, Lee J, Engstrom M, Malarcher A. Pro-tobacco influences and susceptibility to smoking cigarettes among middle and high school students - United States, 2011. J Adolesc Health 2013;52:45-51.

19. Alkhatib MN, Holt RD, Bedi R. Smoking and tooth discolouration: findings from a national cross-sectional study. BMC Public Health 2005;5:27. Disponível em: http://bmcpublic health.biomedcentral.com/articles/10. 1186/1471-2458-5-27

20. Galbiatti AL, Padovani-Junior JA, Maniglia JV, Rodrigues DS, Pavarino EC, Goloni-Bertollo EM. Head and neck cancer: causes, prevention and treatment. Braz J Otorhinolaryngol 2013;79(2):239-47

21. Hashibe M, Brennan P, Benhamou S, Castellsague X, Chen C, Curado MP et al. Alcohol drinking in never users of tobacco, cigarette smoking in never drinkers, and the risk of head and neck cancer: pooled analysis in the International Head and Neck Cancer Epidemiology Consortium. J Natl Cancer Inst 2007;99: 777-89.

22. Madruga CS, Laranjeira R, Caetano R, Pinsky I, Zaleski M, Ferri C. Use of licit and ilicit substances among adolescents in Brazil - A national survey. Addictive Behaviors 2012;37:1171-75.

23. Associação Brasileira de Empresas de Pesquisa-ABEP. Critério de Classificação Econômica do Brasil. Disponível em: http://www.abep.org.

24. Smith DR, Leggat PA. An international review of tobacco smoking among dental students in 19 countries. Int Dent J 2007;57(6):452-8.

25. Andrade APA, Bernardo ACC, Viegas CAA, Ferreira DBL, Gomes TC, Sales MR. Prevalência e características do tabagismo em jovens da Universidade 
de Brasília. J Bras Pneumol 2006;32(1):23-8.

26. Horta RL, Horta BL, Pinheiro RT, Morales B, Strey MN. Tabaco, álcool e outras drogas entre adolescentes em Pelotas, Rio Grande do Sul, Brasil: uma perspectiva de gênero. Cade Saúde Pública 2007;23(4):775-83.

27. Fachini A, Aliane PP, Martinez EZ, Furtado EF. Efficacy of brief alcohol screening intervention for college students (BASICS): a meta-analysis of randomized controlled trials. Subst Abuse Treat Prev Policy 2012;7:40. Disponível em: http://www.ncbi. nlm.nih.gov/pmc/articles/PMC34992 25/.

28. Ramis TR, Mielke GI, Habeyche EC, Oliz MM, Azevedo MR, Hallal PC. Tabagismo e consumo de álcool em estudantes universitários: prevalência e fatores associados. Rev Bras Epidemiologia 2012; (15):376-85.

29. Wagner GA, Stempliuk VA, Zilberman ML, Barroso LP, Andrade AG. Alcohol and drug use among university students: gender differences. Rev Bras Psiquiatr 2007;29(2):123-9.

30. Agaku IT, Ayo-Yusuf OA, Vardavas CI, Connolly G. Predictors and patterns of cigarette and smokeless tobacco use among adolescents in 32 countries, 2007-2011. J Adolesc Health 2014;54:47-53.

\section{ABSTRACT \\ Socioeconomic profile of dental students who use alcohol and tobacco.}

The aim of this study was to evaluate alcohol and tobacco consumption among dental students. A cross-sectional census study was conducted with 300 dental students at the Federal University of Paraná (UFPR). Socioeconomic data, as well as data related to alcohol and smoking consumption were collected through a questionnaire developed specifically for this research. The majority of the studied population was female and mean age was $21 \pm 2.5$ years. A total of $51.3 \%$ used alcohol and $8.4 \%$ used tobacco. Both alcohol $(\mathrm{PR}=1.58 ; 95 \% \mathrm{CI}: 1.09-2.28)$ and tobacco $(\mathrm{PR}=1.72 ; 95 \% \mathrm{CI}: 1.10-2.70)$ use were more prevalent among male students. Both alcohol $(\mathrm{PR}=1.70 ; 95 \% \mathrm{CI}: 1.30-2.21)$ and tobacco $(\mathrm{PR}=1.50 ; 95 \% \mathrm{CI}: 1.10-2.05)$ use were more prevalent among students from higher economic classes in comparison to those from less privileged classes. Alcohol use was $70 \%$ more prevalent among individuals who did not live with their families in comparison to those who lived with their families $(P R=1.70 ; 95 \%$ CI: 1.10-2.65). No association was found between living situation and tobacco. Dental students at UFPR who use alcohol and tobacco have a specific socioeconomic profile. Thus, efforts should be directed toward protecting and raising the awareness of this group.

Descriptors: Students, Dental. Smoking. Alcoholic Beverages.

Correspondência para:

Prof Fabian Calixto Fraiz

e-mail: fabianfraiz@gmail.com

Avenida Prefeito Lothário Meissner, 632

Jardim Botânico

80210-170 - Curitiba - Paraná 